\title{
Experimental verification and validation of a computer model for light-tissue interaction
}

\author{
A E Karsten ${ }^{1,2}$, A Singh ${ }^{1}$, M W H Braun ${ }^{2}$ \\ ${ }^{1}$ Biophotonics Group, National Laser Centre, CSIR, P. O. Box 395, Pretoria, 0001, South Africa \\ ${ }^{2}$ Department of Physics, University of Pretoria, Pretoria, 0002, South Africa
}

\begin{abstract}
Laser light is used frequently in both diagnostics and treatment of patients. For any laser treatment to be effective it is important to deliver the correct dose at the treatment site. Human skin scatters and absorbs laser light in the visible wavelength region which results in a decrease in fluence some distance into the skin. Computer simulations can be used to predict the fluence at the treatment site. Liquid and solid phantoms were prepared and the optical properties were measured. These values were then used as input values to a commercial software package simulating the different layers of skin representing phantoms. The transmission and reflected fractions of the different phantoms were measured with an integrating sphere and compared with the computer simulations. The results showed very good agreement with the measured values and the model can therefore be used with confidence to predict fluence at any treatment site inside the skin.
\end{abstract}

Keywords: laser, light propagation, tissue, phantom

\section{Introduction}

Laser and light treatment modalities in the health and beauty fields are increasing rapidly worldwide. A large number of these treatments need to penetrate through some skin layers before reaching the target treatment area.

It is well reported in literature that the optical properties of human skin tissue influence the absorption and scattering of light through the tissue [1-5]. Absorption is wavelength dependent and is influenced by skin type. Melanin in the skin (present in the epidermis) is responsible for skin colour and absorbs light in the visible and near infrared (near IR) part of the light spectrum. The different skin types (Type I -VI on the Fitzpatrick scale [6]) have different concentrations of melanin. In the South African population all the skin types are represented which poses a challenge in determining the correct dose for laser or light based treatment modalities.

Photodynamic therapy (PDT), a light based treatment for skin cancer, is currently under investigation in various research laboratories. The initial screening of the drug for a specific cancer is normally done on mono cell layers without the inclusion of the outer skin layers that are present during treatment in a clinical setting.

Computer modelling can be an invaluable tool to determine the laser dose that reaches the treatment site. The accuracy of any mathematical model depends on both the accuracy of the input parameters and the assumptions made in the model.

The aim of this work is to determine if the commercial software package, ASAP from Breault Research, for raytracing through turbid media, can be used to determine the fluence $\left(\mathrm{W} / \mathrm{cm}^{-2}\right)$ at a pre-determined depth into the sample. If this can be achieved, the software can then be used to implement different geometries, including tumours in the skin, for the different skin types and accurately predict the fluence at the treatment depth.

A computer model of the interaction of light with skin was developed in the ASAP environment and tested on skin simulating phantoms. The total light transmitted through and reflected off several skin simulating phantoms were measured and compared with the values predicted by the computer model. 


\section{Optical properties}

In this paper the following optical properties will be used:

- Absorption coefficient $\left(\mu_{a}\right)$ : the attenuation of light through a sample due to the absorption of the light.

- Scattering coefficient $\left(\mu_{s}\right)$ : the attenuation of light through a sample due to the scattering of the light in the medium.

- Total attenuation coefficient $\left(\mu_{t}\right): \mu_{t}=\mu_{s}+\mu_{a}$

- Anisotropy $(g)$ : the angular distribution of scatter in the medium $(g=<\cos \theta>)$. The value of $g$ varies between -1 and 1 (backscatter to forward scatter). For $g=0$, the model describes a material with a uniform probability of scattering at all angles, but as $\mathrm{g}$ approaches 1 the distribution becomes highly peaked in the forward direction.

- Angular distribution of light scattered by small particles is described by the HenyeyGreenstein model. This model has been applied to numerous situations, ranging from the scattering of light by biological tissue to scattering by interstellar dust clouds [7]. In this work, the angular distribution of scattered light is given by:

$$
\rho(\theta)=\frac{1}{4 \pi} \frac{1-g^{2}}{\left[1+g^{2}-2 g \cos (\theta)\right]^{3 / 2}}
$$

- Reduced scattering coefficient $\left(\mu_{\mathrm{s}}^{\prime}\right)$ : a parameter combining the scattering coefficient $\left(\mu_{\mathrm{s}}\right)$ and the scatter directionality $(\mathrm{g})$. This is a parameter normally measured in turbid media. $\mu_{s}^{\prime}=(1-g) \mu_{s}$.

According to Star [8], measuring $g$ accurately is very difficult. For most tissue types, including skin, the condition for validity of the diffusion approximation $\left(\mu_{a}<<\mu_{s}^{\prime}\right)$ is satisfied in the visible wavelengths [8]. This means that the precise value of $g$ is not needed for the regions far from the boundaries and the light source. For the calculation of the light distribution in skin it is then sufficient to measure only $\mu_{a}$ and $\mu_{s}^{\prime}$ accurately while knowing the value of $g$ approximately. The sensitivity of the total transmission through the sample, to changes in $g$ while keeping $\mu_{s}^{\prime}\left(\mu_{s}^{\prime}=\right.$ $\left.(1-g) \mu_{s}\right)$ constant, was tested for a specific solid phantom. Results showed very small changes and are described in more detail in the Optimisation section.

\section{Materials and methods}

Phantoms

Two types of phantoms were prepared, namely solid phantoms and liquid phantoms. Liquid phantoms are easy to prepare, but they have limitations in terms of durability/lifetime and only simple geometries are possible. Solid phantoms, which are much more cumbersome to prepare, have a longer lifetime and can be used to model multi layered structures, simulating the different layers of skin. Liquid phantoms are prepared from Intralipid, which is used widely to simulate skin and the optical properties are well documented in literature [4, 9-12]. For the solid phantoms the optical properties need to be measured.

\section{Solid phantoms}

Solid phantoms were prepared according to the recipe of Firbank [13]. Scattering particles (TiO particles, $<25 \mathrm{~nm}$ particle size, density $=3.9 \mathrm{~g} / \mathrm{mL}$ at $25^{\circ} \mathrm{C}$ ) and absorbing particles (Carbon Black) were added to an optically clear resin base (Akasel) and mixed for 5 minutes. Thereafter the hardener (Aka-cure slow, Akasel) was added to the mixture and stirred at 5 minute intervals, at least 3 times to prevent settling of particles and to eliminate air bubbles. The mixture was poured into plastic containers with a diameter of $30 \mathrm{~mm}$. The mixture was allowed to cure at room temperature under a fume hood for 24 hours. 
The cured solid phantoms were cut into discs with a thickness of approximately $2 \mathrm{~mm}$ and a diameter of $30 \mathrm{~mm}$. The amount of $\mathrm{TiO}$ was varied while the carbon black concentration was kept constant. The ratios (per weight) were 133:2:31000 (TiO:carbon-black:resin) for sample A and 56.8:2:31000 for sample B. A two layered phantom was prepared by pouring one phantom mixture into the mould allowing it to cure and then pouring the new phantom mix onto the existing solid phantom. Both $\mu_{\mathrm{a}}$ and $\mu_{\mathrm{s}}^{\prime}$ were measured with the integrating sphere (IS) system described below [14].

\section{Liquid phantoms}

The scattering properties of the skin can be simulated by Intralipid, a white, fat based emulsion with spherical particles (mean diameter of $0.4 \mu \mathrm{m}$ ). [15] Intralipid phantoms were prepared by mixing 20\% Intralipid (IL) (Sigma Aldrich, lot \# 028K0740) and black ink. The black ink is used to simulate the absorption in skin. In the wavelength region $600-800 \mathrm{~nm}$, the light scattering in tissue far exceeds the absorption in the tissue and therefore the diffusion approximation can be used and the reduced scattering coefficient $\left(\mu_{\mathrm{s}}^{\prime}\right)$ is sufficient to describe all the tissue scattering properties $[8,16]$.

The black ink was diluted in distilled water and the absorption coefficient $\left(\mu_{\mathrm{a}}\right)$ of the black ink was measured with a HeNe laser at a wavelength of $632.8 \mathrm{~nm}$, using Beer-Lambert law:

$$
I=I_{0} \exp ^{-\mu_{t} d}
$$

with $\quad I$ : measured laser intensity after the sample

$I_{0}$ : initial laser intensity

$d$ : optical pathlength

$\mu_{t}: \quad$ total attenuation $\left(\mu_{t}=\mu_{s}+\mu_{a}\right)$

For the ink diluted in water it is assumed that there is no scattering, only absorption and therefore the Beer-Lambert law can be written as:

$$
I=I_{0} \exp ^{-\mu_{a} d}
$$

The ink solution and the IL solution were then mixed in a ratio of $1: 1$ before putting the liquid in the cuvette (Figure1) for measurements.

The optical properties of Intralipid-10\% are well documented for a HeNe laser (wavelength of $632.8 \mathrm{~nm}$ [9-12]). In the model, $g$ was calculated using the relationship between $g$ and $\lambda$ in the work by Straveren [11]. The reduced scattering coefficient was calculated using the expression from the work of Michielsen [10]. His work expresses the reduced scattering coefficient as a function of the concentration of Intralipid. Most other papers only report the values for Intralipid$10 \%$. The reduced scattering coefficient of the IL was calculated by [10]:

$$
\begin{gathered}
\mu_{\mathrm{s}}^{\prime}(\lambda=633 \mathrm{~nm})=1.104 \mathrm{~mm}^{-1} \times(\text { IL conc) } \\
\mu_{\mathrm{a}}=0.15 \times 10^{-2} \mathrm{~mm}^{-1} \mathrm{x} \text { (IL conc) }
\end{gathered}
$$

the value of $\mu_{\mathrm{a}}$ can be disregarded for the Intralipid- $10 \%$.

The anisotropy was calculated by [11]:

where $\alpha=0.58 \mu \mathrm{m}^{-1}$

$$
\mathrm{g}=1.1-\alpha \lambda
$$

\section{Cuvettes}

The cuvette, used for the IL phantoms, was prepared by gluing two transparent glass microscope slides together with a black plastic spacer in-between (Figure 1). The optical path length, $d$ (distance between the two microscope slides) is $1.47 \mathrm{~mm}$ and the diameter in the black plastic is $30 \mathrm{~mm}$. 


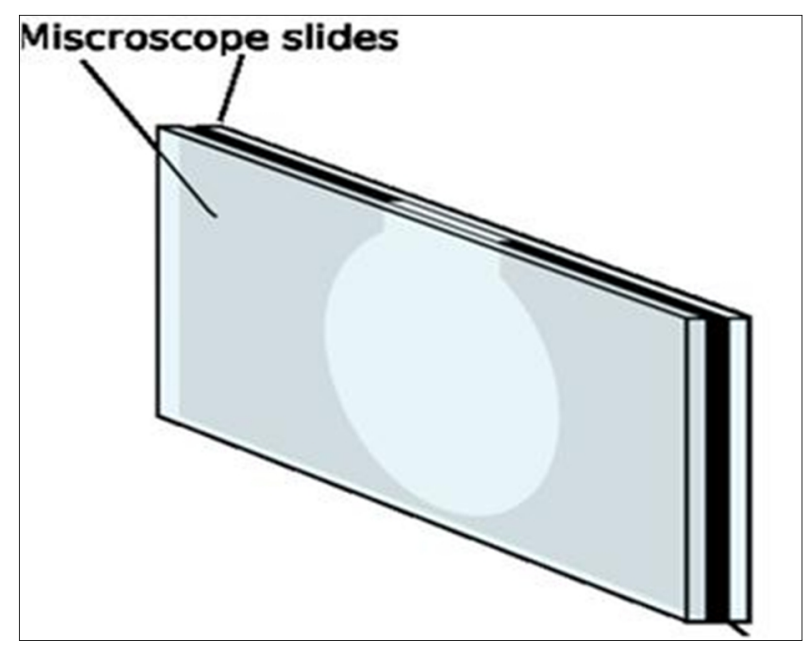

Figure 1: Schematic of the cuvettes for the Intralapid phantoms.

\section{Integrating sphere measurements}

The measurements on the integrating sphere (IS) system (Figure 2) were used to extract the absorption and reduced scattering coefficients for each solid phantom using the multiple regression method on a well calibrated model $[17,18]$. These values were used as input parameters for the computer model.

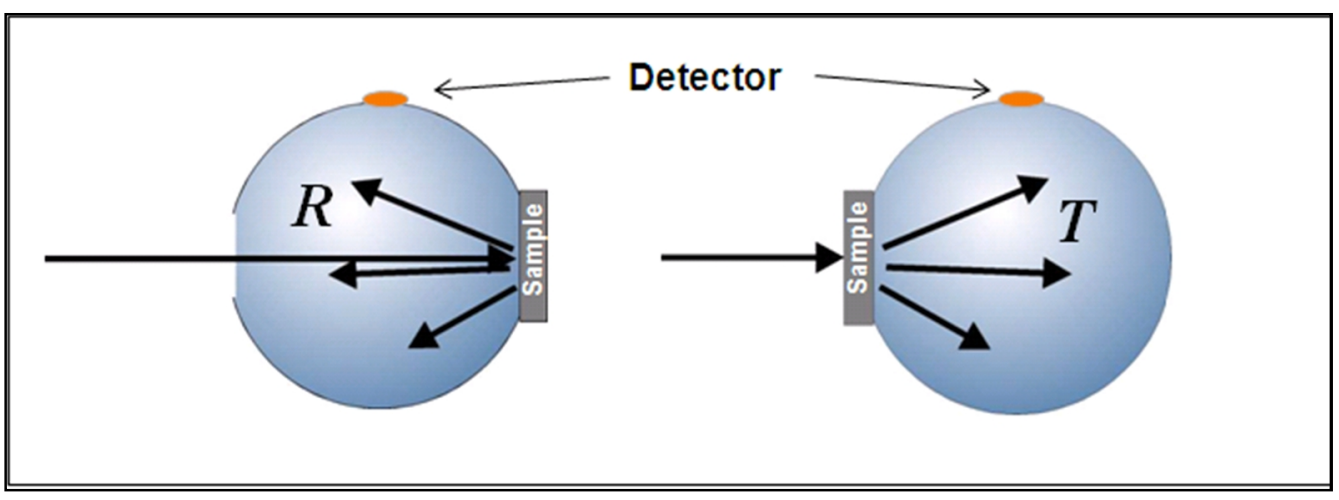

Figure 2: Integrating sphere system for reflection and transmission measurements.

The IS system comprised of a $7.4 \mathrm{~mW}$ HeNe laser ( $\lambda=632.8 \mathrm{~nm}$, JDS Uniphase laser) collimated to a beam diameter of 2-3 $\mathrm{mm}$ at the entrance and exit ports of the sphere (Labsphere, diameter $203.2 \mathrm{~mm}$ ). Diffuse transmittance and reflectance and total transmittance for each sample was taken in triplicate on a spectrometer (Ocean Optics USB4000).

\section{Computer simulations}

Raytracing software is commonly available and is mainly based on the Monte Carlo Multi-Layered (MCML) programs developed by Jacques [19]. The software is downloadable online [20]. These programs use the Monte Carlo raytracing techniques where "photons" or "rays" are traced through a medium with pre-defined absorption and scattering properties, until the photons are completely absorbed or leave the medium through the boundaries of the model.

The Advanced Systems Analysis Program (ASAP $®$ ) from Breault Research, a commercial software package, with the advantage of allowing the user to easily make geometry changes to a tissue model, was used for this work. Different tissue geometries and tumours (e.g. layered 
structures, spheres and uneven surfaces) can be simulated in the software. Due to a lack of scientific publications making use of this software for modelling the interaction of light with skin tissue, this paper tries to verify the model with the use of custom made tissue simulating phantoms that can be measured in an optical laboratory. Before any laser treatment can progress to clinical trials, the safety of the treatment needs to be established. This model will be able to calculate the fluence at any given distance into the skin to ensure that safety standards and treatment parameters are met. The final aim of this work will be to model the absorption of laser light as it passes through skin tissue in the presence of a small tumour and the drug used in photodynamic therapy, a form of cancer therapy.

In the model the geometry of the phantom was described. A light source was created and traced though the sample. ASAP is in essence a non-sequential raytracing software package, which means that a ray interacts with the optical surfaces as they are encountered. Rays are allowed to split due to Fresnel reflections. ASAP ceases to trace a ray if its flux has become too low. In ASAP a ray is a purely geometrical concept, it is basically a vector that simulates radiative transfer. Each ray has an individual power or flux that contributes to the total flux of the source. [21]

In order to describe the optical system in ASAP, the geometry must be known as well as the absorption coefficient $\left(\mu_{a}\right)$, scattering coefficient $\left(\mu_{s}\right)$, anisotropy $(g)$ and refractive index $(n)$ of all the materials in the system.

A three layer slab model was created, but the number of layers can be changed as required. Two different geometries were created, the one presenting the liquid phantom or cuvette, and the other presenting a one or two layer solid phantom disc. A collimated light source with a diameter of $1 \mathrm{~mm}$ at a wavelength of $633 \mathrm{~nm}$ was used. A semi-spherical detector was placed behind the light source to collect all the back-scattered light and a round detector plate was placed $0.2 \mathrm{~mm}$ behind the last phantom surface in order to measure the transmitted light. These two detectors only serve as evaluation surfaces and are not part of the calculations in the models. 3.1 million rays were traced through the system. For evaluations the phantom was divided into three dimensional pixels (or voxels) of about $0.1 \times 0.1 \times 0.1 \mathrm{~mm}^{3}$. The phantom was divided into thin slices $(0.1 \mathrm{~mm}$ thick) in the Z-direction and the number of slices depended on the thickness of the sample. The absorption in each of the voxels is recorded. The total absorption in each layer is calculated as well as the absorption in the sides of the phantom (ring part of the disc). The transmitted light is measured with the circular detector just after the last surface of the phantom and the back-scattered light is measured with the semi-spherical detector behind the light source (Figure 3). In this model, the angular distribution of scatter is given by the Henyey-Greenstein phase function [7], discussed under Optical Properties.

The geometric parameters and optical properties of the IL phantoms are given in Tables 1 and 2. Six different IL phantoms were prepared. All the optical properties were kept the same except for the absorption coefficient.

Table 1: Geometric parameters for the IL phantoms.

\begin{tabular}{|l|c|c|c|c|}
\hline Layer & Width $(\mathbf{m m})$ & Length $(\mathbf{m m})$ & Diameter $(\mathbf{m m})$ & Thickness $(\mathbf{m m})$ \\
\hline Glass & 39 & 77 & - & 1.09 \\
\hline IL & - & - & 30 & 1.47 \\
\hline
\end{tabular}




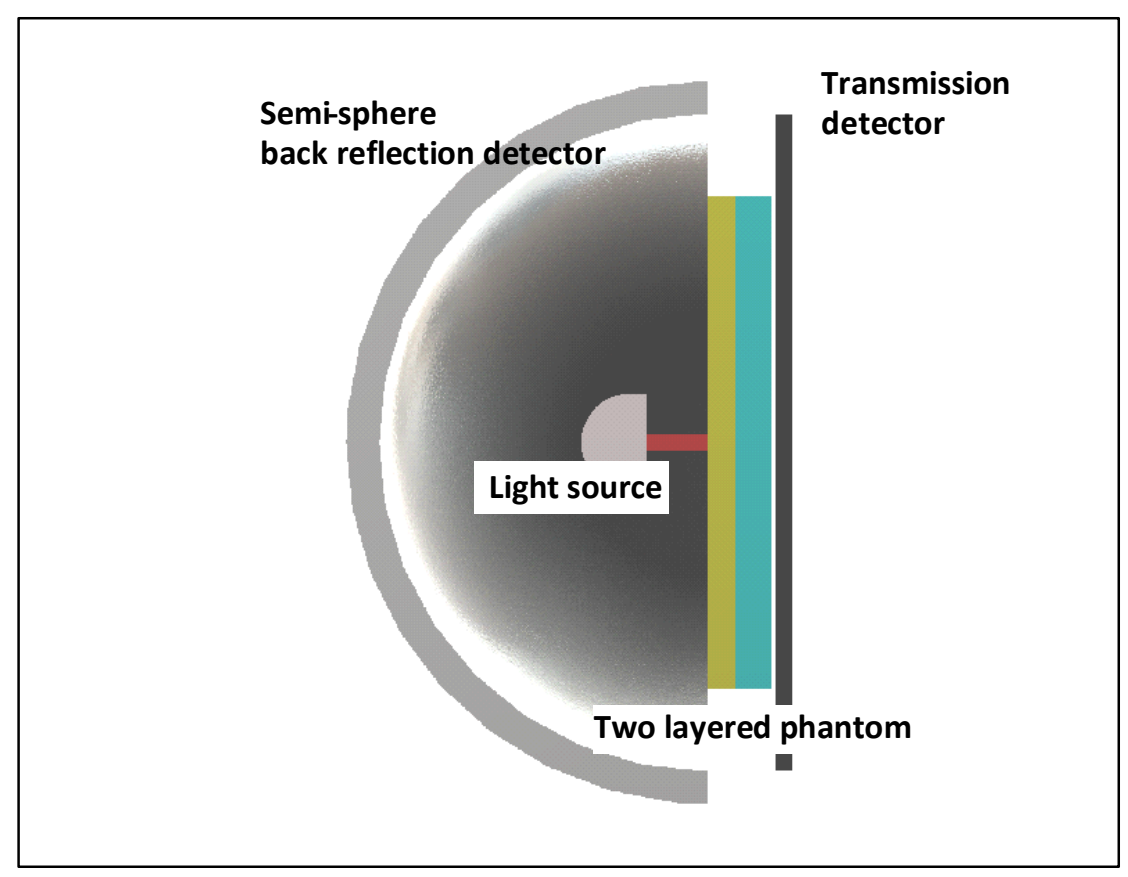

Figure 3: Side view of the computer model of a two-layered solid phantom. The semi-sphere back reflector detector as well as the circular transmission detector plate are visible.

Table 2: Optical parameters for the IL phantoms used in the model.

\begin{tabular}{|l|l|l|l|c|}
\hline Sample & $\boldsymbol{\mu}_{\boldsymbol{a}}\left(\mathbf{m m}^{-\mathbf{1}}\right)$ & $\boldsymbol{\mu}_{\boldsymbol{s}}\left(\mathbf{m m}^{-\mathbf{1}}\right)$ & $\boldsymbol{g}$ & $\boldsymbol{n}$ \\
\hline Glass & - & - & - & 1.5 \\
\hline ILA & 0.0000 & & & \\
\cline { 1 - 2 } ILB & 0.0515 & & & \\
\cline { 1 - 2 } ILC & 0.0816 & \multirow{3}{*}{39.897} & 0.7329 & 1.33 \\
\cline { 1 - 2 } ILD & 0.1665 & & & \\
\cline { 1 - 2 } ILE & 0.2350 & & & \\
\cline { 1 - 2 } ILF & 0.4565 & & & \\
\hline
\end{tabular}

Table 3: Optical parameters for the solid phantoms. Parameters for the A and B samples are given. Sample $\mathrm{C}$ used the $\mu_{a}$ and $\mu_{s}$ values from $\mathrm{A}$ and $\mathrm{B}$ respectively for the 2 layers in the phantom.

\begin{tabular}{|c|c|c|c|c|c|}
\hline Sample & $\boldsymbol{\mu}_{\boldsymbol{a}}\left(\mathbf{m m}^{-1}\right)$ & $\boldsymbol{\mu}_{\boldsymbol{s}}\left(\mathbf{m m}^{-\mathbf{1}}\right)$ & $\boldsymbol{d}(\mathbf{m m})$ & $\boldsymbol{n}$ & $\boldsymbol{g}$ \\
\hline $\mathbf{A}$ & 0.268 & 10.38 & 1.66 & 1.4 & 0.79 \\
\hline B & 0.138 & 4.85 & 2.3 & 1.4 & 0.79 \\
\hline $\mathbf{C}$ & $\begin{array}{c}\text { A and B } \\
\text { values }\end{array}$ & $\begin{array}{c}\text { A and B } \\
\text { values }\end{array}$ & $\begin{array}{c}3.9 \\
(1.7+2.2)\end{array}$ & 1.4 & 0.79 \\
\hline
\end{tabular}

In all the calculations the step size was kept at $0.001 \mathrm{~mm}$, which is small compared to $\mu_{t}^{-1}$, the average mean free path length of a photon [22].

For each sample the absorption in each layer or slice is calculated. The flux through the last layer in the sample is also calculated. The total transmitted power of the light through the sample (as a percentage of the input power) is measured with the IS system and with the detector plate in the model. The back-scattered light as a percentage of the input power, was also measured for all the phantoms using the IS system. In the model the backscattered light is "measured" on the semisphere detector. 


\section{Optimisation}

Sensitivity of the model was tested on the solid phantom B. The following parameters were evaluated: effect of slice thickness, number of voxels, number of rays and sensitivity to $\mathrm{g}$. The results are summarised in Table 4.

Table 4: Optimisation results showing the total absorption though the phantom, absorption in the last layer, the transmitted power and the standard deviation (Stdev) of the data.

\begin{tabular}{|c|c|c|c|c|c|c|}
\hline \multirow{2}{*}{ Parameter } & \multicolumn{2}{|c|}{ Total Absorption } & \multicolumn{2}{c|}{$\begin{array}{c}\text { Absorption in last } \\
\text { layer }\end{array}$} & \multicolumn{2}{c|}{ Transmitted } \\
\cline { 2 - 7 } & Mean & Stdev & Mean & Stdev & Mean & Stdev \\
\hline $\begin{array}{c}\text { Slice thickness (0.209- } \\
\mathbf{0 . 0 3 5} \mathbf{~ m m )}\end{array}$ & $5.83 \mathrm{E}-1$ & $1.58 \mathrm{E}-3$ & - & - & $1.73 \mathrm{E}-1$ & 0 \\
\hline \# voxels (100-400) & $5.85 \mathrm{E}-1$ & $1.66 \mathrm{E}-4$ & $1.10 \mathrm{E}-2$ & $3.99 \mathrm{E}-4$ & $1.73 \mathrm{E}-1$ & 0 \\
\hline $\begin{array}{c}\text { \# rays (3.1M, 19.6M, } \\
\mathbf{7 8 . 5 M )}\end{array}$ & $5.85 \mathrm{E}-1$ & 0 & $1.12 \mathrm{E}-2$ & 0 & $1.73 \mathrm{E}-1$ & 0 \\
\hline $\boldsymbol{\mu}_{\boldsymbol{s}}$ and $\boldsymbol{g}$ & $1.05 \mathrm{E}-2$ & $5.66 \mathrm{E}-4$ & $4.16 \mathrm{E}-1$ & $1.13 \mathrm{E}-3$ & $1.74 \mathrm{E}-1$ & $1.18 \mathrm{E}-3$ \\
\hline
\end{tabular}

- Slice thickness:

○ The effect of the slice thickness (normally around $0.1 \mathrm{~mm}$ ) was evaluated to establish if that has an effect on the total transmission through the sample. For the sample thickness of $2.3 \mathrm{~mm}$ the number of slices varied between 11 and 66 resulting in slice thicknesses between $0.209 \mathrm{~mm}$ and $0.035 \mathrm{~mm}$.

○ Conclusion: Changes in the slice thickness does not significantly affect the output parameters. The model was standardised on a slice thickness of $0.1 \mathrm{~mm}$.

- Number of voxels:

o Changing the number of voxels in the $\mathrm{X}$ and $\mathrm{Y}$ directions from 100 to 400 resulted in small changes in the total absorption through the model and the absorption in the last layer.

- Conclusion: 300 voxels were used in the $\mathrm{X}$ and $\mathrm{Y}$ direction to correspond to the $0.1 \mathrm{~mm}$ intervals of the Z-direction.

- Number of rays:

- The number of rays traced through the sample was tested for 3.1, 19.5 and 78.5 million. The changes in the number of rays did not influence the total transmission or absorption results.

- Conclusion: Simulations were all done with 3.1 million rays because of the reduced computing time required. Imaging simulations were done with the higher number of rays.

- Sensitivity for the model to $\mu_{s}$ and $g$ :

○ The product $\left(\mu_{s}^{\prime}=(1-g) \mu_{s}=1.019 \mathrm{~mm}^{-1}\right)$ was kept constant and the value of $g$ varied from $0.6-0.8$ with corresponding changes in $\mu_{s}$. The total absorption, transmitted light and the absorption in the last layer were relatively constant when 3.1 million rays were traced.

○ Conclusion: The model is insensitive to changes in $g$, while keeping the product $\left(\mu_{s}^{\prime}=(1-g) \mu_{s}\right)$ constant [8].

Experimental verifications

The IS system described earlier was used to measure the transmitted power as well as the back scattered power of each sample. The back plane (last surface) of each sample was also imaged with a CCD camera. The imaging experimental setup is shown in Figure 4. A Spiricon camera with detector dimensions $7.1 \mathrm{~mm}$ x $5.4 \mathrm{~mm}$ was used. An external lens was placed between the phantom and the camera and a magnification $(\mathrm{M}=\mathrm{v} / \mathrm{u})$ of 0.5 was used. Absorbing neutral density filters from Thorlabs were put in front of the detector to protect the camera from damage. 


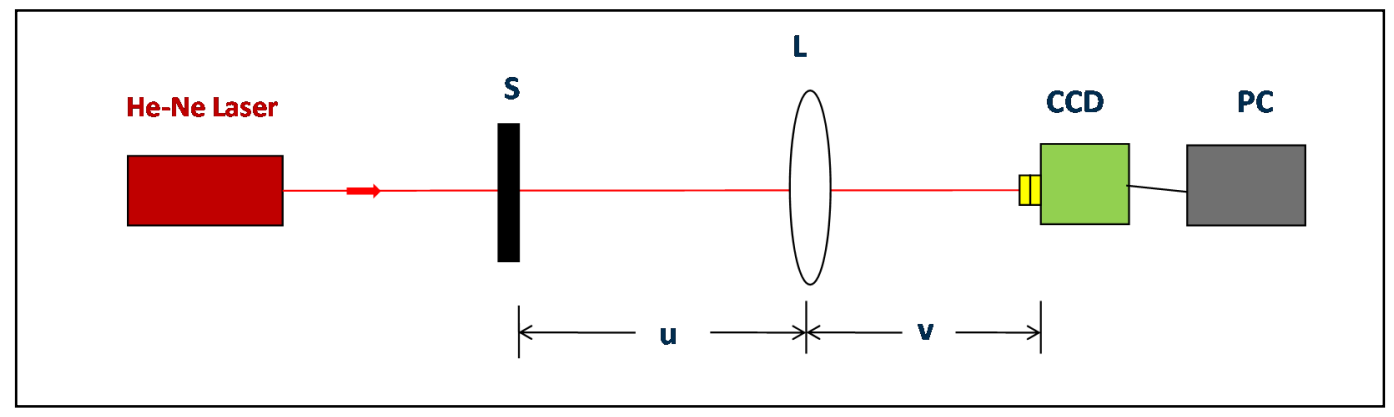

Figure 4: Experimental setup: $\mathrm{S}$ (Sample), $\mathrm{L}$ (Lens $\mathrm{f}=150 \mathrm{~mm}, \mathrm{D}=50.8 \mathrm{~mm}$ ), CCD (Camera), PC (Computer), u (Object distance $=450 \mathrm{~mm}), \mathrm{v}($ Image distance $=225 \mathrm{~mm}), \mathrm{F}=$ filters, $\mathrm{M}(\mathrm{v} / \mathrm{u})=0.5$, HeNe Laser power $=9 \mathrm{~mW}$.

\section{Results}

The results for the absorption and transmission measurement comparisons are evaluated first and then the results for the imaging experiments on the IL samples.

Transmitted and reflected power

Results comparing the reflective (\%Refl) and transmission (\%Trans) measurements on the IS with the calculations through the model as well as the total absorption (\%Abs) through the model are presented in Tables 5 and 6.

\section{Solid phantoms}

Table 5: Comparisons between the model and measured values for the solid phantoms.

\begin{tabular}{|c|c|c|c|c|c|}
\hline Sample & \%Abs & \%Trans (IS) & \%Trans (Sim) & \%Refl (IS) & \%Refl (Sim) \\
\hline A & 65 & 9.9 & 8.8 & 27.4 & 25 \\
\hline B & 59 & 19.3 & 17 & 25.3 & 24 \\
\hline C & 70 & 3.0 & 2.7 & 26.6 & 26 \\
\hline
\end{tabular}

All three samples gave good agreement when comparing the light transmitted through the phantom and the backscattered light calculated by the computer model and measured on the IS system.

\section{Liquid phantoms}

Table 6: Comparisons between the model and measured values for the liquid phantoms.

\begin{tabular}{|l|c|c|c|c|c|}
\hline Sample & \%Abs & \%Trans (IS) & \%Trans (Sim) & \%Refl (IS) & \%Refl (Sim) \\
\hline ILA & 0 & 12 & 7.6 & 72 & 63.6 \\
\hline ILB & 7.4 & 7 & 6.7 & 61 & 60.6 \\
\hline ILC & 13.1 & 6.6 & 6.2 & 59.0 & 59.1 \\
\hline ILD & 21.1 & 2 & 5.0 & 55 & 54.8 \\
\hline ILE & 27.9 & 1.9 & 4.2 & 54 & 51.8 \\
\hline ILF & 44.2 & 0.29 & 2.4 & 39 & 44.0 \\
\hline
\end{tabular}

The agreement between the measured and calculated values for the transmission and reflectance for the pure IL sample (ILA) and the sample with a high absorption coefficient (ILF) are not as good for the solid samples. However the agreement between the IS measurements and the simulations on the phantoms containing both IL and a lower concentration black ink (samples ILB-ILE) was good. This is also the area of interest for most of the modelling work when applying the work to skin transmissions. 


\section{CCD images}

The back surfaces of the IL phantoms were imaged on a CCD camera as shown in Figure 4. The laser power was $9 \mathrm{~mW}$. For consistency the same filters (Optical density 1 and 2) were used for each phantom. The images were converted to contour images and the minimum and maximum values for all three images were used to derive at 4 equally spaced contour values (Figure 5(a)). The fluence plot (images) of the last layer in the model was also converted to contour plots (Figure 5(b)) to compare with the CCD image contour plots.

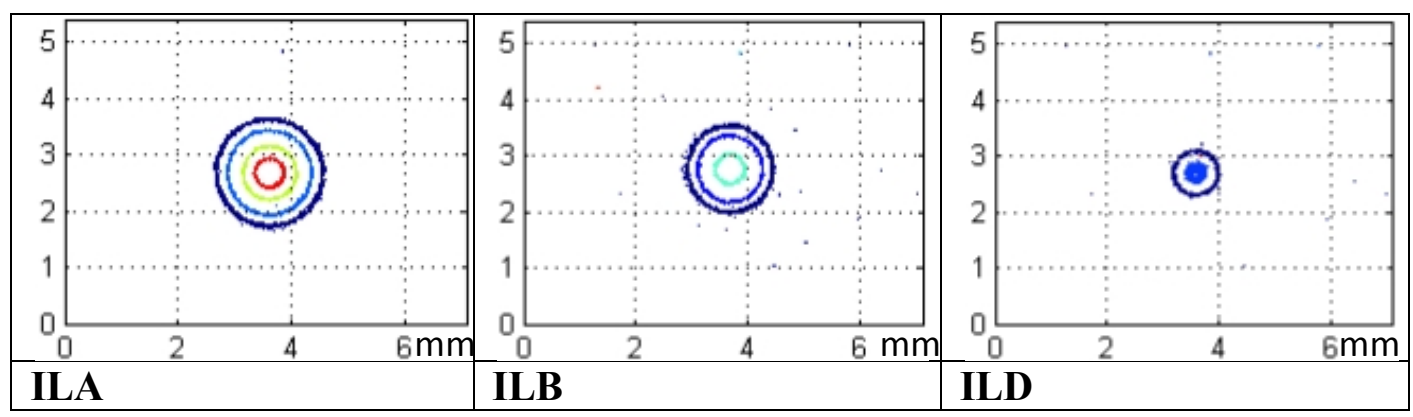

Figure 5a: Fluence contour plots from the CCD images of the back surface of the liquid phantoms.

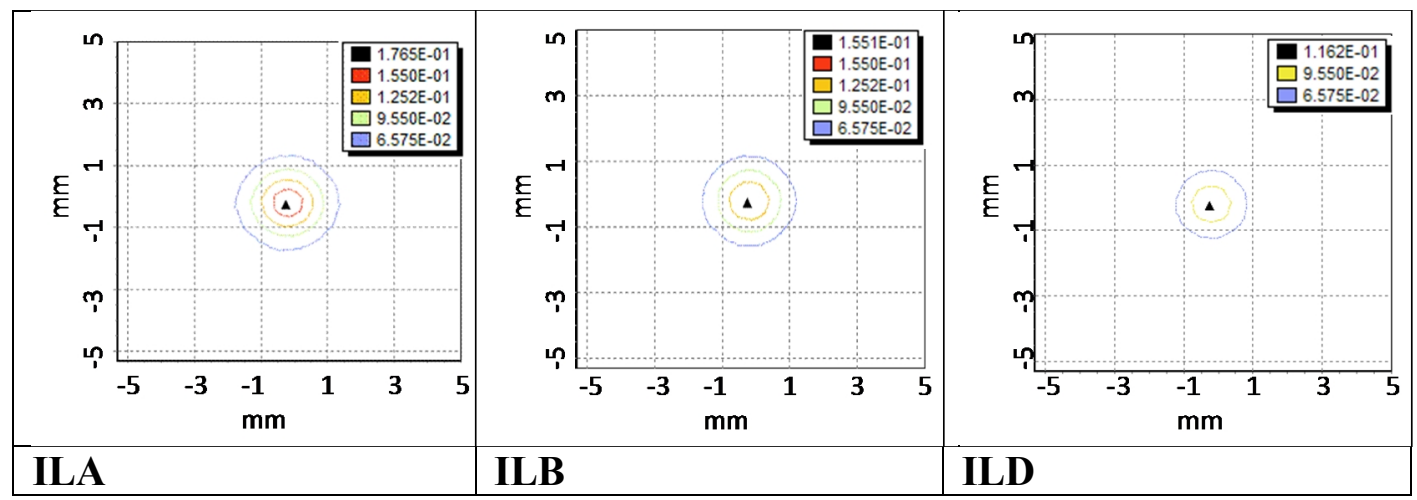

Figure 5b: Fluence contour plots from the calculated fluence through the last layer in the IL phantom model.

There are very good similarities between the CCD image contour plots and those of the computer model in terms of the decreasing spot size of the beam with increased absorption in the sample.

\section{Discussions and conclusion}

The results in Table 5 showed good agreement between the calculated and measured values for the solid phantoms. There are small differences in the values for the solid phantom, that may be attributed to the error margins $(<5 \%)$ that exists in the IS calibration model $[17,18]$.

Phantoms consisting of IL and ink are known to simulate the optical properties of skin. The $\mu_{\mathrm{a}}$ values used are comparable to the values that are expected to be present in the South African population. The parameters used for the IL simulations were based on the published data. The error margins for these values are not known. This, combined with errors introduced in the IS system when measuring low transmissions (typical error margin measures are $\pm 5 \%$ ) may explain the differences between the higher absorption samples in Table 6 . These results imply that the model is not valid for the extremes in terms of no absorption and high absorption. This will impact on the validity of the model. At the present the model seems to be valid for $\mu_{\mathrm{a}}$ values between 0.05 and $0.26 \mathrm{~mm}^{-1}$ for the HeNe wavelength of $632.8 \mathrm{~nm}$. The validity of the model outside these boundaries still needs further investigations. The model was only tested for wavelength of $632.8 \mathrm{~nm}$. 
Qualitatively, the contour results also showed good agreement in terms of trends. Filters used in the experiments influence the 'size' of the beam in the image. The main value of the images is to show that the trend in terms of the beam size is the same in the phantoms as in the model.

The advantage of modelling software is the ability of the software to calculate the light fluence at any position inside the model/skin. If the front surface values (back-scattered) and the end values (transmission) of the model agree, it is a fair assumption to make that the values in the middle part are also correct. In the model an input power of $1 \mathrm{~mW}$ is used. In practice the laser power will be much more, but the important part is to use the absorbing, scattering and transmission fractions to predict the values for higher input powers. The model was also tested for an input power of $10 \mathrm{~mW}$ which resulted in higher absolute values (10X), but the same fractions.

The data presented here indicates that the computer model can be used to accurately calculate the light fluence at layers inside the sample. In the next phase more complicated geometries and drugs can be added to the model to simulate cancerous lesions.

\section{Acknowledgements}

The authors would like to acknowledge Mr Bafana Moya for the work in the preparation and optical laboratories.

\section{References}

1. Tuchin V (2000) Tissue Optics: Light Scattering Methods and Instruments for Medical Diagnostics. SPIE Press, pp 3-108

2. Drakaki E, Psycharakis S, Makropoulou M, Serafetinides AA (2005) Optical properties and chromophore concentration measurements in tissue-like phantoms. Optics Communications 254: 40-51

3. Jacques SL (2010) How tissue optics affect dosimetry of photodynamic therapy. Journal of Biomedical Optics. doi: 10.1117/1.3494561

4. Star WM, Marijnissen JPA, van Gemert MJC (1988) Light dosimetry in optical phantoms and in tissues: I. Multiple flux and transport theory. Phys. Med. Biol. Vol 3 No 4 437-454

5. Grossweiner LI (1997) PDT light dosimetry revisited. Journal of Photochemistry and Photobiology B: Biology 38 pp 258-268

6. Agache P, Humbert P (2004) Measuring the skin. Springer-Verlag, ISBN 3-540-01771-2, pp473-474

7. Henyey L, Greenstein J (1994) Diffuse radiation in the galaxy. Astrophys. Journal 93, pp $70-83$

8. Star WM (1997) Light dosimetry in vivo. Phys. Med. Biol. 42 pp 763-787

9. Flock S, Jaques S, Wilson B (1992) Optical properties of intralipid A phantom medium for light propagation study. Lasers in Medicine and Surgery, Volume 2, 1992, pp510-519

10. Michielsen K, De Raedt H, Przeslawski J, Garcia N (1998) Computer simulation of timeresolved optical imaging of objects hidden in turbid media. Physics Reports 304 pp 89144

11. van Staveren HJ, Moes CJM, van Marie J, Prahl SA, van Gemert MJC (1991) Light scattering in Intralipid-10\% in the wavelength range of 400-1 $100 \mathrm{~nm}$. Applied Optics, Vol. 30, No. 31 pp 4507-4514 
12. Choukeife JE, L'Huillier JP (1999) Measurements of Scattering Effects Within Tissuelike Media at Two Wavelengths of $632.8 \mathrm{~nm}$ and $680 \mathrm{~nm}$. Lasers Med Sci, 14,pp 286296

13. Firbank M, Delpy DT (1993) A design for a stable and reproducible phantom for use in near infra-red imaging and spectroscopy. Phys. Med. Bid. 38 pp 847-853

14. Singh A, Karsten AE, Mputle I, Chetty A, Naidoo K (2009) Determination of the optical properties of PNIPAAm gels used in biological applications, Proc. SPIE 7373, 737317 doi:10.1117/12.831882

15. Das BB, Liu F, Alfano RR (1997) Time-resolved fluorescence and photon migration studies in biomedical and model random media. Rep. Prog Phys. 60 pp 227-292.

16. Dimofte A, Finlay JC, Zhu TC (2005) A method for determination of the absorption and scattering properties interstitially in turbid media. Phys. Med. Biol. 50 pp 2291-2311

17. Dam JS, Dalgaard P, Fabricius PE, Andersson-Engels S (2000) Multiple polynomial regression method for determination of biomedical optical properties from integrating sphere measurements. Appl. Opt 39 pp 1202-1209

18. Singh A, Karsten AE, Dam, J. S (2008) Robustness and accuracy of the calibration model for the determination of the optical properties of chicken skin. Proceedings of the International Conference of the World Association of Laser Therapy, Sun City, South Africa, pp 165-169, (CD ISBN 978-88-7587-471-1)]

19. Jacques SL (2008) Modeling tissue optics using Monte Carlo modeling: a tutorial. Optical Interactions with Tissue and Cells XIX, edited by Steven L. Jacques, William P. Roach, Robert J. Thomas, Proc. of SPIE Vol. 6854, 68540T. 1605-7422/08/\$18 · doi: 10.1117/12.776997 Proc. of SPIE Vol. 6854 68540T-1

20. http://omlc.ogi.edu/software/mc/

21. ASAP Technical guide Radiometric analysis http://www.breault.com/resources/kbasePDF/brotg0909 radiometry.pdf

22. Prahl SA, Keijzer M, Jacques SL, Welch AJ (1989) A Monte Carlo Model of Light Propagation in Tissue. SPIE Institute Series Vol. IS 5 pp 102-111 